ARTICLE

\title{
Local destruction of superconductivity by non-magnetic impurities in mesoscopic iron-based superconductors
}

Jun $\mathrm{Li}^{1,2,3}$, Min Ji ${ }^{2,3}$, Tobias Schwarz ${ }^{4}$, Xiaoxing $\mathrm{Ke}^{5}$, Gustaaf Van Tendeloo ${ }^{5}$, Jie Yuan ${ }^{3,6}$, Paulo J. Pereira ${ }^{7}$, Ya Huang ${ }^{2,3}$, Gufei Zhang ${ }^{1}$, Hai-Luke Feng 3,8, Ya-Hua Yuan 3,8, Takeshi Hatano ${ }^{3}$, Reinhold Kleiner ${ }^{4}$, Dieter Koelle ${ }^{4}$, Liviu F. Chibotaru', Kazunari Yamaura 3,8, Hua-Bing Wang ${ }^{2,3}$, Pei-Heng Wu2 ${ }^{2}$, Eiji Takayama-Muromachi, ${ }^{8,9}$, Johan Vanacken ${ }^{1} \&$ Victor V. Moshchalkov ${ }^{1}$

The determination of the pairing symmetry is one of the most crucial issues for the iron-based superconductors, for which various scenarios are discussed controversially. Non-magnetic impurity substitution is one of the most promising approaches to address the issue, because the pair-breaking mechanism from the non-magnetic impurities should be different for various models. Previous substitution experiments demonstrated that the non-magnetic zinc can suppress the superconductivity of various iron-based superconductors. Here we demonstrate the local destruction of superconductivity by non-magnetic zinc impurities in $\mathrm{Ba}_{0.5} \mathrm{~K}_{0.5} \mathrm{Fe}_{2} \mathrm{As}_{2}$ by exploring phase-slip phenomena in a mesoscopic structure with $119 \times 102 \mathrm{~nm}^{2}$ cross-section. The impurities suppress superconductivity in a three-dimensional 'Swiss cheese'-like pattern with in-plane and out-of-plane characteristic lengths slightly below $\sim 1.34 \mathrm{~nm}$. This causes the superconducting order parameter to vary along abundant narrow channels with effective cross-section of a few square nanometres. The local destruction of superconductivity can be related to Cooper pair breaking by non-magnetic impurities.

\footnotetext{
${ }^{1}$ INPAC - Institute for Nanoscale Physics and Chemistry, KU Leuven, Celestijnenlaan 200D, Leuven B-3001, Belgium. ${ }^{2}$ Research Institute of Superconductor Electronics, Nanjing University, Nanjing 210093, China. ${ }^{3}$ National Institute for Materials Science, 1-1 Namiki, Tsukuba 305-0044, Japan. ${ }^{4}$ Physikalisches Institut-Experimentalphysik II and Center for Collective Quantum Phenomena in LISA+, Universität Tübingen, Auf der Morgenstelle 14, Tübingen D-72076, Germany. ${ }^{5}$ Electron Microscopy for Materials Research (EMAT), University of Antwerp, Groenenborgerlaan 171, Antwerp B-2020, Belgium. ${ }^{6}$ Condensed Matter Physics, Institute of Physics, Chinese Academy of Sciences, Beijing 100190, China. ${ }^{7}$ Division of Quantum and Physical Chemistry and INPAC-Institute for Nanoscale Physics and Chemistry, KU Leuven, Celestijnenlaan 200F, Leuven B-3001, Belgium. ${ }^{8}$ Graduate School of Chemical Science and Engineering, Hokkaido University, Hokkaido 060-0810, Japan. ${ }^{9}$ WPI-MANA, National Institute for Materials Science, 1-1 Namiki, Tsukuba 305-0044, Japan. Correspondence and requests for materials should be addressed to J.L. (email: junli@nju.edu.cn), H.-B.W. (email: hbwang1000@gmail.com) or V.V.M. (email: Victor.Moshchalkov@fys.kuleuven.be).
} 
F or the newly discovered high- $T_{\mathrm{c}}$ Fe-based superconductors ${ }^{1}$, it is essential to elucidate the pairing symmetry of the superconducting wave function ${ }^{2}$, for which the multi-gapped $s$-wave is generally acceptable. Possible candidates include the unconventional $s \pm$-wave with sign-reversal ${ }^{3}$ and the conventional $s_{++}$-wave without sign-reversal ${ }^{4}$. According to Anderson's theorem ${ }^{5-8}$, a few at $\%$ of non-magnetic impurities can act as strong scattering centres and dramatically suppress superconductivity by pair breaking in the case of an anisotropic gap, for example, in a $d$-wave or $^{9} \pm$ wave $^{2,3}$ superconductor. Our previous experiments demonstrated that non-magnetic $\mathrm{Zn}$ impurities can suppress the transition temperature $T_{\mathrm{c}}$ of the 122-type Fe-based superconductors ${ }^{10}$, while the magnitude of the $T_{\mathrm{c}}$ suppression is lower than expected for the $s \pm$-wave scenario $^{4,11}$. On the basis of these results, recent theoretical studies proposed that the suppression of superconductivity could be attributed to various effects apart from pair breaking, such as localization ${ }^{12}$ or disorder ${ }^{13,14}$. Furthermore, orbital fluctuations, as the possible origin of the $s_{++}$state, could be suppressed by lifting the orbital degeneracy near impurity atoms ${ }^{15}$, which would also lead to a reduction in $T_{c}$. Therefore, the corresponding theoretical calculation of $T_{c}$ suppression ${ }^{10,11}$ cannot support the pair breaking by non-magnetic impurities as the responsible mechanism directly, which requires further understanding on the role of $\mathrm{Zn}$.

The impurity ions of $\mathrm{Zn}^{2}+$ behave as spinless centres, which may have induced moments of $s=2$ on the Fe sites (the 'Kondo-hole problem'). Nuclear magnetic resonance (NMR) can probe nuclei coupled to the superconducting $\mathrm{Fe}_{2} \mathrm{X}_{2}$ planes to yield information on the local magnetic structure. Kitagawa et al. ${ }^{16}$ studied the $\mathrm{Zn}$-substituted $\mathrm{LaFeAsO}_{0.85}$ polycrystal using ${ }^{75} \mathrm{As}$ and ${ }^{139} \mathrm{La}$ NMR and nuclear quadrupole resonance, and found that the suppression of superconductivity by $\mathrm{Zn}$ is not due to the change of the normal-state properties, but due to a strong non-magnetic pair-breaking effect on superconductivity. On a local-scale of suppression, the $\mathrm{Zn}$ ions can exclude the supercurrent of an unconventionally gapped superconductor within an area of $\pi \xi_{a b}^{2}$, which results in a two-dimensional (2D) 'Swiss cheese'-like supercurrent distribution; here $\xi_{a b}$ is the in-plane coherence length of the superconductor. This model was proposed based on the in-plane Zn-doping studies of cuprate superconductors by scanning tunnelling spectroscopy $(\text { STM })^{17}$ and muon spin relaxation experiments ${ }^{18,19}$, while such experiments on the iron pnictides are still in progress ${ }^{20}$. Since the Fe-based 122 compounds possess nearly isotropic properties $^{21}$, the superconducting order parameter $\psi$ can also fluctuate along the $c$ axis. Therefore, it is essential to study both the in-plane and out-of-plane effects of $\mathrm{Zn}$ ions on local superconductivity, which can hardly be observed by STM or muon spin relaxation.

Local destruction of superconductivity by $\mathrm{Zn}$ impurities will result in a considerable suppression of the superconducting volume fraction. Thus, it is promising to study this behaviour in mesoscopic samples, in which local effects should be more pronounced $^{22}$. For a one-dimensional (1D) superconducting system, it has been proposed that $\psi$ may be spatially or temporally modulated along its length at finite temperature $T$. In this case, resistive behaviour is induced by thermally activated events, which cause $\psi$ to shrink to zero and slip its phase by $2 \pi$. Such a process is denoted as thermally activated phase-slip ${ }^{23-30}$. Strictly speaking, it is hard to obtain a $1 \mathrm{D}$ superconductor experimentally with a diameter less than the characteristic length $\xi$. A quasi-1D system with corresponding dimensions being smaller than $\sqrt{2} \pi \xi$, is more feasible ${ }^{25-28}$. For low- $T_{\mathrm{c}}$ superconductors with coherence lengths $\xi(T)$ up to a few micrometres, the $1 \mathrm{D}$ regime has been reached experimentally via micro- and/or nanopatterning techniques, and phase-slip processes have been observed ${ }^{31-34}$. However, this approach seems to be rather challenging for the high- $T_{\mathrm{c}}$ superconductors where $\xi$ is extremely small ( $\sim 2 \mathrm{~nm}$ or less) and comparable to the size of just one unit cell for both cuprates and the Fe-based superconductors ${ }^{1,10}$. Surprisingly, recent measurements on a $\mathrm{YBaCu}_{3} \mathrm{O}_{7-\delta}$ (YBCO) nanobridges $\left(50 \times 100 \mathrm{~nm}^{2}\right.$ in cross-section) demonstrated a pronounced thermal phase-slip behaviour ${ }^{35}$. Since cuprate nanostructures are quite susceptible to degradation induced by chemical and thermal instability during nanopatterning, the observed phase-slip behaviour may have been dominated by inhomogeneities in the YBCO nanobridges. In contrast to this, for the $\mathrm{Ba}_{0.5} \mathrm{~K}_{0.5} \mathrm{Fe}_{2} \mathrm{As}_{2}$ (BK) system, one can hardly observe any degradation by the patterning process ${ }^{36}$. This makes the system quite promising for studies of the impact of impurities on the superconducting properties in BK nanostructures.

Here we present an approach to study the effects of local destruction of superconductivity by introducing non-magnetic $\mathrm{Zn}$ impurities into iron arsenide $\mathrm{Ba}_{0.5} \mathrm{~K}_{0.5} \mathrm{Fe}_{1.94} \mathrm{Zn}_{0.06} \mathrm{As}_{2}$ (BKZn) mesoscopic superconducting structures. Phase-slip behaviour was observed in nanobridges of width $W$ and thickness $h$ with cross-section areas $A=W \times h$ down to $119 \times 102 \mathrm{~nm}^{2}$. We propose that $\mathrm{Zn}$ suppresses superconductivity in a three-dimensional (3D) 'Swiss cheese'-like fashion, where $\psi$ fluctuates along abundant narrow channels, which are supposed to be a few nanometres wide, close to the size of $\xi$.

\section{Results}

Transport properties. Figure 1a shows a scanning electron microscopy image of the nanobridge BKZn-N1 (width $W=119$ $\mathrm{nm}$, thickness $h=102 \mathrm{~nm}$ ). The resistance $R$ versus $T$ curves of nanobridges BKZn-N1 and BKZn-N2 $(W=290 \mathrm{~nm}, h=315 \mathrm{~nm})$, and of the microbridge BKZn-M1 $(W=2000 \mathrm{~nm}, h=373 \mathrm{~nm})$ are given in Fig. 1b. The microbridge BKZn-M1 exhibits a sharp superconducting transition, indicating bulk behaviour. On the other hand, the $R(T)$ curves of the nanobridges show several steps, which are particularly pronounced for BKZn-N1 with the smallest cross-section $A$. The transition width $\Delta T_{\mathrm{c}} \sim 11 \mathrm{~K}$ of $\mathrm{BKZn-N1}$ is also larger than that of the other bridges (for example, $\Delta T_{\mathrm{c}} \sim 3 \mathrm{~K}$ for BKZn-N2). Similar steps and a broadened $\Delta T_{\mathrm{c}}$ in the $R(T)$ curves were frequently observed in low- $T_{\mathrm{c}}$ nanowires $31-34$ and high- $T_{c}$ YBCO nanobridges ${ }^{35}$, and were explained by thermally activated phase-slips.

Figure 2a shows the current-voltage characteristics (IVCs) of the $\mathrm{Zn}$-doped nanobridge $\mathrm{BKZn-N1}$ taken at $T=2-25 \mathrm{~K}$. On increasing the bias current from zero, the sample switches to an intermediate resistive state (for example, at a critical current $I_{\mathrm{c}}=0.64 \mathrm{~mA}$ at $T=2 \mathrm{~K}$ ). By further increasing $I$, a second jump to the normal state appears. When the current is swept down, the sample retraps from the normal to the intermediate resistive regime at a retrapping current $I_{\mathrm{r}}$ and finally to the superconducting state after one or two intermediate states. The steps in the IVCs and the $R(T)$ curves exhibit characteristics typical for phase-slips. The pronounced hysteresis on the IVCs can be attributed to Joule heating. The instantaneous dissipation affects the local temperature of the mesoscopic system and results in an increased probability for thermal activation ${ }^{27,37}$. According to Tinkham's theory ${ }^{24}$, the phase-slips can move in a homogeneous wire system (like vortices in a clean crystal), and the phase-slip occurs at a relatively higher current due to the larger critical current, where more heat will be generated to switch the wire into the normal state immediately after the occurrence of a single phase-slip. For inhomogeneous wires, however, a phase-slip can occur and it can be pinned at a weak 

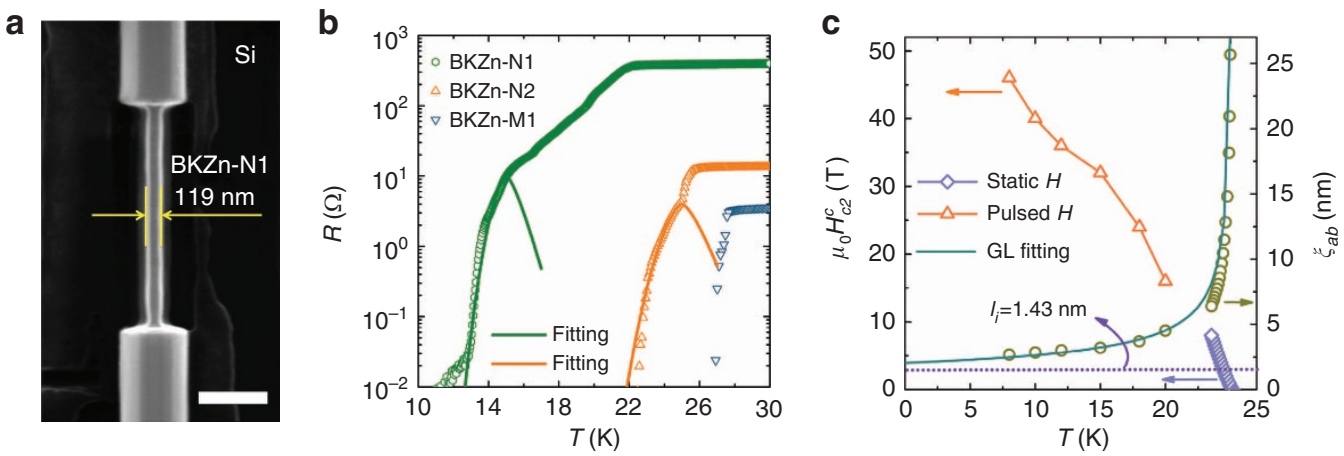

Figure 1 | Image of the nanobridge and transport properties. (a) Scanning electron microscopy image of nanobridge BKZn-N1. Scale bar, 500 nm. (b) $R(T)$ curves measured in zero magnetic field for nanobridges BKZn-N1, BKZn-N2 and microbridge BKZn-M1, which reveal different cross-sectional area (see, for example, Table 1). The bias current for each sample was $10 \mu \mathrm{A}$. Open symbols are experimental data, and solid lines are fitting results from the thermal activated phase-slip model proposed by Little ${ }^{23}$. (c) $\mu_{0} H_{c 2}^{2}(T)$ and $\xi_{a b}(T)$ measured on microbridge BKZn-M1. $\mu_{0} H_{c 2}^{2}(T)$ is extracted from the resistive transition points at $90 \%$ of the normal state resistivity $\rho_{n}$, as determined from a Physical Properties Measurement System in static fields ( $<9 \mathrm{~T}$, see Supplementary Fig. 3) and from a pulsed field set-up up to $52 \mathrm{~T}$ (Supplementary Fig. 5). $\xi_{a b}(T)$ is estimated from the Ginzburg-Landau formula for an anisotropic 3D superconductor $\xi_{a b}(T)=\sqrt{\Phi_{0} / 2 \pi H_{c 2}^{c}(T)}$ (ref. 21); $\Phi_{0}$ is the magnetic flux quantum. We also estimated $\xi_{a b}(T)$ by the Ginzburg-Landau relation $\xi(T)=\xi_{0} / \sqrt{\left(1-T / T_{\mathrm{c}}\right)}$ as shown by the dark cyan line. The horizontal violet dotted line shows the mean distance $l_{i}$ between neighbouring $\mathrm{Zn}$ ions.
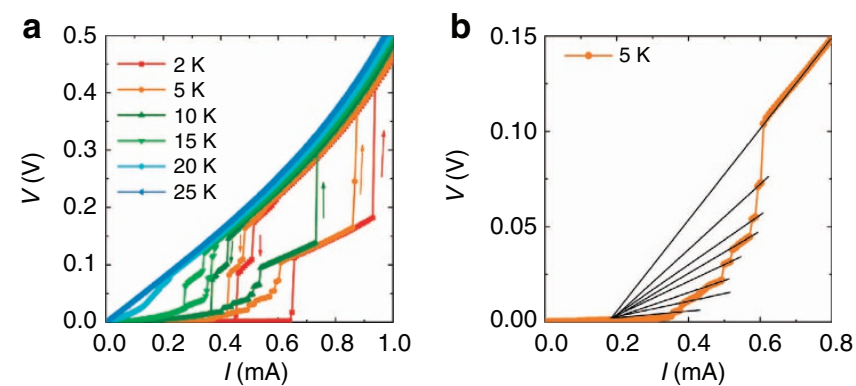

Figure 2 | current-voltage characteristics (IVCs) of nanobridge BKZn-N1. (a) current-voltage characteristics (IVCs) of nanobridge BKZn-N1 measured at different $T$ in zero magnetic field. Arrows indicate bias current sweep directions. (b) Enlarged view for sweep-up IVC at $5 \mathrm{~K}$. Once extrapolating each successive branch linearly, all branches intersect at $V=0$, representing a phase-slip centre as described by Tinkham ${ }^{24}$.

link. Such a phase-slip would be acting at a lower current and thus can exist without overheating the whole wire. For the undoped BK nanobridges, however, IVCs demonstrated switching from the superconducting to the voltage state with absence of any intermediate state (Supplementary Fig. 2).

Figure $2 \mathrm{~b}$ shows an enlarged view of the sweep-up IVC at $5 \mathrm{~K}$. Once extrapolating each successive branch linearly, all branches intersect at $V=0$, representing a typical phase-slip behaviour as described by Tinkham ${ }^{24}$. Each step in the IVC indicates the appearance (somewhere along the nanobridge) of an additional similar localized resistance centre, where the time-average pair chemical potential $\bar{\mu}_{p}$ suffers a discontinuous step-like increment, $\Delta \bar{\mu}_{p}$. Such a centre is usually considered as a phaseslip centre. We also simulated a IVC by applying time-dependent Ginzburg-Landau theory on a 1D system (see Supplementary Note 2 and Supplementary Fig. 9). Comparing the theoretical study with the experimental curves, we conclude that the hysteretic and stairlike behaviour presented in the experimental IVC curves are watermark indications for phase-slip centres.

In Fig. 3, we show the differential resistance $\mathrm{d} V / \mathrm{d} I(I, T)$ for the nanobridge BKZn-N1; Fig. 3a shows data taken by sweeping up the bias current and Fig. $3 b$ shows data for the sweep-down branches of the IVCs. The red points indicate the largest values for $\mathrm{d} V / \mathrm{d} I$, corresponding to the switching currents. For the sweep-up data, we detect at $T=2 \mathrm{~K}$ two intermediate resistive states, which correspond to two avalanche processes of phase-slip. For $T>2 \mathrm{~K}$, more intermediate resistive states are observed, indicating several phase-slip entrances. For the sweep-down branches, we find two major switching steps, as indicated by the two distinct red lines in Fig. $3 b$.

Coherence length. Considering the general condition for the appearance of phase-slips, the cross-sectional area should be compared with $\sqrt{2} \pi \xi$. $\xi(T)$ can be evaluated from the upper critical field $H_{c 2}$ as $\xi_{a b}(T)=\sqrt{\Phi_{0} / 2 \pi H_{c 2}^{c}(T)}$ and $\xi_{c}(T)=\Phi_{0} / 2 \pi H_{c 2}^{a b}(T) \xi_{a b}(T)$ (ref. 21). We measured $H_{c 2}^{c}$ by using static fields up to $\mu_{0} H=8 \mathrm{~T}$ and pulsed fields up to $52 \mathrm{~T}$, applied in the $c$ direction; the results are shown in Fig. 1c together with the corresponding $\xi_{a b}$. We also extrapolated $\xi$ to $T=0$ by using the Ginzburg-Landau expression $\xi(T)=\xi_{0} / \sqrt{\left(1-T / T_{c}\right)}$. This yields $\xi_{a b}(0)=2.05 \mathrm{~nm}$ and $\xi_{c}(0)=1.20 \mathrm{~nm}$, where the latter was determined from d.c. field measurements (Supplementary Figs 3 and 4$)$. The values for $\xi_{a b}(0)$ and $\xi_{c}(0)$ are two orders of magnitude smaller than either the width or the thickness of our nanobridges; this suggests that the dimensions of the nanobridges are too large to allow for the generation of any phase-slips. However, since $\mathrm{Zn}$ induces a local destruction of superconductivity, the effective superconducting regions are likely to be shrunk into narrow channels having a relatively small effective cross-section $A^{*}$ within the nanobridges.

To access $A^{*}$, we fit the $R(T)$ curves by using the thermally activated phase-slip theory proposed by Little ${ }^{23}$. We treat the nanobridge as a quasi-1D system, where a phase-slip passes over a free-energy barrier $\Delta F$ proportional to the cross-sectional area, $R_{\mathrm{PS}}(T)=R_{\mathrm{n}} \exp \left(-\Delta F / k_{\mathrm{B}} T\right)$ (refs $\left.31-34\right)$. Here $R_{\mathrm{n}}$ is the normal resistance, and $\Delta F$ is given by $\Delta F=0.83 k_{\mathrm{B}} T_{\mathrm{c}} \frac{R_{\mathrm{q}} A^{*}}{\rho \xi_{0}}\left(1-\frac{T}{T_{\mathrm{c}}}\right)^{3 / 2}$, where, $R_{\mathrm{q}}=6.45 \mathrm{k} \Omega$ is the superconducting quantum resistance and $\rho_{0}$ is the resistivity (we take the residual resistivity $\rho_{0}=49.98 \mu \Omega \mathrm{cm}$ from results of BKZn-M1). The fitting of $R_{\mathrm{PS}}(T)$ to the $R(T)$ curves is shown in Fig. 1b. From these fits, we obtain $A^{*}=8.26$ and $455.27 \mathrm{~nm}^{2}$ for BKZn-N1 and BKZn-N2, respectively. For BKZn-N1, the obtained $A^{*}$ is three orders of magnitude smaller than the geometric cross-section $A$ of the 

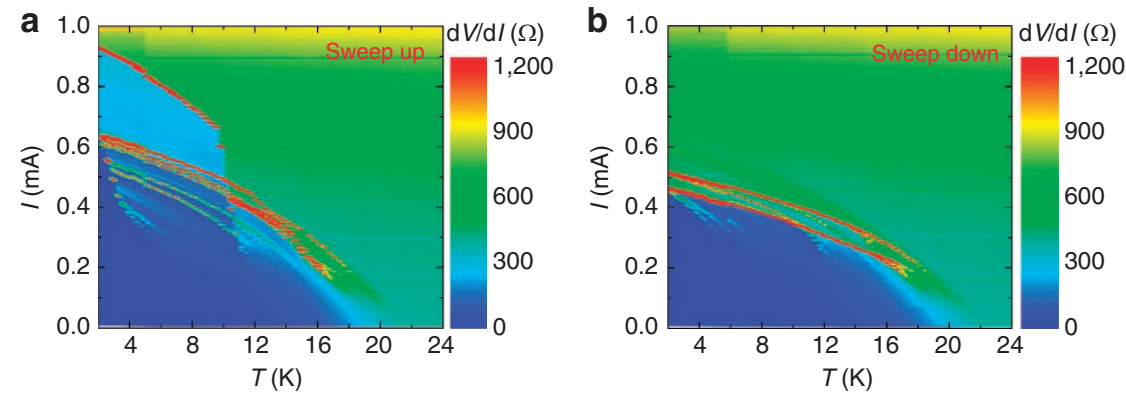

Figure 3 | Differential resistance of the nanobridge BKZn-N1. Bias current / and temperature T-dependent differential resistance dV/d/ at zero magnetic field. The data were collected from (a) sweep-up and (b) sweep-down branches. Red points indicate the critical currents at which large voltage jumps appear.

a

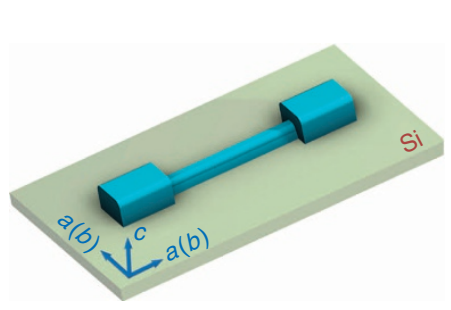

b

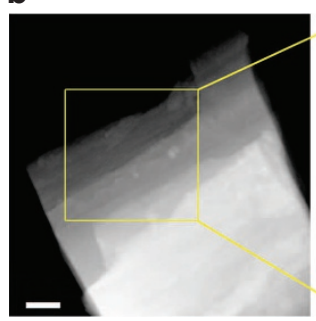

c

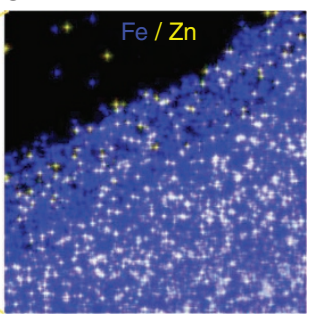

d

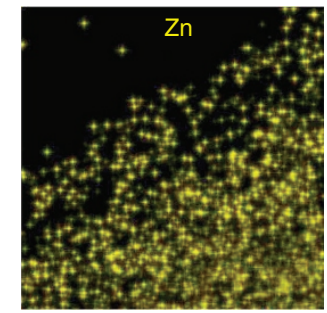

e

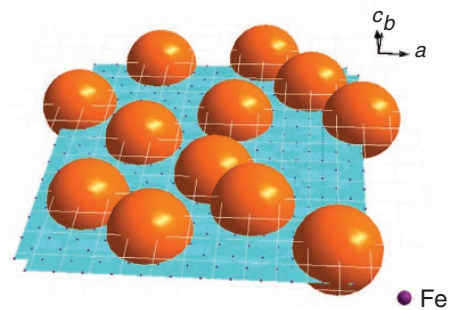

f

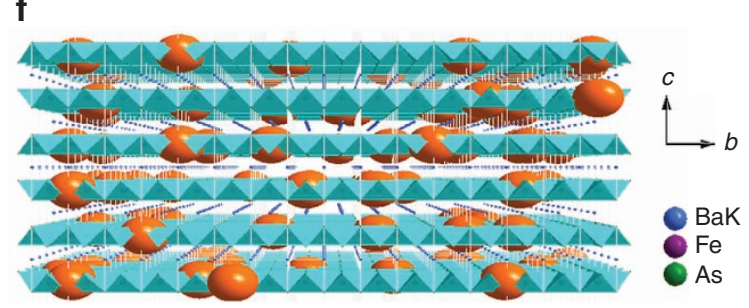

Figure 4 | Swiss cheese model. (a) Transport measurement scheme along a nanobridge. The current flows along the ab plane. (b) HAADF-STEM image of a BKZn flake, where the crystal was detected along the $c$ axis. Scale bar, $50 \mathrm{~nm}$. (c,d) STEM-XEDS mapping for Zn/Fe and Zn distributions, respectively, within the area indicated in $\mathbf{b}$. (e,f) Schematic representation of the 2D and 3D 'Swiss cheese' models, respectively. The yellow oblate spheroid corresponds to the non-superconducting regions centred on $\mathrm{Zn}$ ions with an equatorial length $\xi_{a b}$ and a polar length $\xi_{c}$.

nanobridge, while it is less than the value of $2 \pi^{2} \xi_{a b}^{2}$. For $\mathrm{BKZn}-\mathrm{N} 2, A^{*}$ is relatively large but still two orders of magnitude smaller than its cross-section $A$. We note that the phase-slip phenomenon can only appear within a narrow temperature interval close to $T_{\mathrm{c}}$, where $\xi$ is relatively large, as shown in Fig. 1c.

Superconducting order parameter fluctuation. We can conclude that $\mathrm{Zn}$ plays a significant role in reducing the cross-section of superconducting channels and for the observation of the phase-slip phenomenon, that is, $\mathrm{Zn}$ suppresses superconductivity locally. Considering fluctuations of the superconducting order parameter $\psi$, the influence of $\mathrm{Zn}$ on the $1 \mathrm{D}$ or quasi-1D high- $T_{\mathrm{c}}$ unconventional superconductors can induce two different types of $\psi$ variations, in-plane and out-of-plane.

First of all, since the 122-type superconductors possess an anisotropic-layered structure, the Cooper pairs prefer to reside within the $\mathrm{Fe}_{2} \mathrm{As}_{2}$ superconducting planes. Previous X-ray and in-plane resistivity analysis indicated that the $\mathrm{Zn}$ ions were substituted on the Fe sites of $\mathrm{Ba}_{0.5} \mathrm{~K}_{0.5} \mathrm{Fe}_{2-x} \mathrm{Zn}_{x} \mathrm{As}_{2}$ (ref. 10). Thus, the Cooper pairs can be broken and the supercurrent will be excluded from a $\mathrm{Zn}$-centred area of $\pi \xi_{a b}^{2}$ within the planes, vitalizing the so-called $2 \mathrm{D}$ 'Swiss cheese' model ${ }^{18,19}$ as shown in
Fig. 4e. To explore the distribution of $\mathrm{Zn}$ ions within the $a b$ plane of BKZn crystals, we performed high-angle annular dark-field scanning transmission electron microscopy (HAADF-STEM), as shown in Fig. 4b, together with STEM-energy-dispersive X-ray spectroscopy (STEM-XEDS) mapping as shown in Fig. 4c,d. The $\mathrm{Zn}$ ions were found to be homogeneously distributed within the $a b$ plane of the crystal, without any indication for phase separation. Since only 3 at\% of Fe ions were substituted by $\mathrm{Zn}$ in our crystals, we presume the idealized situation that the $\mathrm{Zn}$ ions are homogenously distributed within the $\mathrm{Fe}_{2} \mathrm{As}_{2}$ layers. Thus, a mean distance $l_{i}$ between $\mathrm{Zn}$ ions can be estimated as $\sim 1.60 \mathrm{~nm}$ using $l_{i}=r / \sqrt{x}$, where $x$ is the Zn-doping level, and $r=2.77 \AA$ is the distance between the neighbouring $\mathrm{Fe}$ ions. $l_{i}$ is significantly smaller than $\xi_{a b}(T)$, especially, near $T_{c}$ (see Fig. 1c). As a result, it seems very likely, that the non-superconducting regions induced by $\mathrm{Zn}$ impurities inhibit superconductivity almost within the whole crystal. In addition, we estimate the in-plane non-superconducting volume fraction $\eta=x \pi \xi_{0}^{2} /(\sqrt{2} a)^{2}=2.35$, where $a$ is the lattice constant. Thus, it seems that superconductivity can hardly survive in crystals with such doping level of $\mathrm{Zn}$. However, previous experiments indicated that superconductivity of $\mathrm{BK}$ can be strongly resistant against $\mathrm{Zn}$ impurities up to $x=10$ at\% (ref. 36). 


\begin{tabular}{|llrrc|}
\hline \multicolumn{4}{|c|}{ Table 1 | Dimensions of all micro- and nanobridges. } \\
Samples & \multicolumn{1}{c|}{ Materials } & $\boldsymbol{W}(\mathbf{n m})$ & $\boldsymbol{L}(\mathbf{n m})$ & $\boldsymbol{h}(\mathbf{n m})$ \\
\hline $\mathrm{BK}-\mathrm{N} 1$ & $\mathrm{Ba}_{0.5} \mathrm{~K}_{0.5} \mathrm{Fe}_{2} \mathrm{As}_{2}$ & 340 & 402 & 105 \\
$\mathrm{BKZn}-\mathrm{N} 1$ & $\mathrm{Ba}_{0.5} \mathrm{~K}_{0.5} \mathrm{Fe}_{1.94} \mathrm{Zn}_{0.06} \mathrm{As}_{2}$ & 119 & 1,452 & 102 \\
$\mathrm{BKZn}-\mathrm{N} 2$ & $\mathrm{Ba}_{0.5} \mathrm{~K}_{0.5} \mathrm{Fe}_{1.94} \mathrm{Zn}_{0.06} \mathrm{As}_{2}$ & 290 & 1,558 & 315 \\
$\mathrm{BKZn-M1}$ & $\mathrm{Ba}_{0.5} \mathrm{~K}_{0.5} \mathrm{Fe}_{1.94} \mathrm{Zn}_{0.06} \mathrm{As}_{2}$ & 2,000 & 10,000 & 373 \\
\hline
\end{tabular}

To investigate the local destruction area of Zn, STM experiments will be the most promising way. But, unfortunately, the STM study on the $\mathrm{Zn}$-doping effect on Fe-based superconductors is still under work. The main challenge for the STM experiments is due to the technical difficulty in high-quality single-crystal fabrication and in situ cleaving technique. Zhu et al. ${ }^{38}$ have calculated a single nonmagnetic impurity behaviour in the $(\mathrm{K}, \mathrm{Tl}) \mathrm{Fe}_{x} \mathrm{Se}_{2}$ superconductors for various models. The impurity-induced resonance state was found to exist only for the $d_{x^{2}-y^{2}}$-wave pairing state, but not for the others like $s \pm$. Besides, the bound-state peak in the local density of states occurs at a non-zero energy for Fe-based systems even in the unitary limit, indicating an opposite situation from the cuprate systems. On the basis of the experimental results on $\mathrm{Zn}$ doping, Chen et al. ${ }^{39}$ suggested that the in-plane $\xi$ for the case of a $s \pm$ paring symmetry should be as short as two lattice sites, namely, $\xi_{\Delta} \sim 0.55 \mathrm{~nm}$. Such a short screening length was attributed mainly to the strong local Coulomb repulsion $U$, which acts on the charges. We note that the corresponding $\eta$ is quite low, 0.17 for $x=3$ at $\%$ and $\xi_{\Delta} \sim 0.55 \mathrm{~nm}$. In this case, the $\psi$ fluctuations could be strong enough to induce the motion of phase-slips, thus inhibiting the generation of thermally activated phase-slips ${ }^{24}$. Therefore, we argue that the characteristic length should be slightly $<1.34 \mathrm{~nm}$, which is estimated from the boundary condition $\eta=1$.

Considering the out-of-plane variations of $\psi$, since $\xi_{c}$ perpendicular to the plane $\left(\xi_{c}(0)=1.20 \mathrm{~nm}\right)$ of the crystal is larger than the distance between each superconducting plane $(t=0.665$ $\mathrm{nm}$ (ref. 10)), the amplitude of $\psi$ can be easily strong enough for Josephson coupling between the layers ${ }^{40}$, resulting in a weakly anisotropic behaviour as discussed before ${ }^{21,36} . \xi_{c}(0)$ of $\mathrm{Zn}$-doped samples is larger than $t=0.666 \mathrm{~nm}$ as well ${ }^{10}$, and particularly, $\xi_{c}(T)$ is increasing with increasing $T$. However, with Zn doping, the non-superconducting islands around the $\mathrm{Zn}$ ions can break the superconducting structure symmetry along the $c$ axis, leading to an inhomogeneous $3 \mathrm{D}$ structure, for which we propose a $3 \mathrm{D}$ 'Swiss cheese' model as shown in Fig. $4 \mathrm{f}$, namely, stacks of $2 \mathrm{D}$ 'Swiss cheese' separated by $\mathrm{Ba} / \mathrm{K}$ barrier layers. The presence of non-superconducting islands acts as additional barriers enhancing the distance between the adjacent superconducting layers and weakening the Josephson coupling along the $c$ axis. Consequently, we may assume that $\psi$ develops along abundant narrow superconducting channels within the nanobridges, whose $A^{*}$ should be dramatically smaller than the cross-sectional area.

\section{Discussion}

On the basis of the 3D 'Swiss cheese' model, the phase-slip phenomenon could be observed in a bulk crystal once the $\mathrm{Zn}$ impurity is homogenously distributed within the crystal. However, we can hardly perform transport measurements on a bulk crystal because of the dramatically higher value of $I_{\mathrm{c}}$. Instead, we studied microbridges with $2 \mu \mathrm{m}$ width and thicknesses ranging from 49.2 to $479.5 \mathrm{~nm}$. Phase-slip was found for microbridges with thickness up to $415.5 \mathrm{~nm}$ within a narrow temperature region just below $T_{\mathrm{c}}$ (see Supplementary Fig. 6 and Supplementary Note 1). For thinner bridges, however, phase-slip phenomenon was enhanced quite substantially. Indeed, one can hardly synthesize an ideal crystal with $\mathrm{Zn}$ ions distributed within the $\mathrm{Fe}_{2} \mathrm{As}_{2}$ plane homogenously. The sheet resistance $\left(R_{\square}\right)$ of the $\mathrm{Zn}$-doped crystal is $R_{\square}=\rho_{n} / t \approx 211.7 \Omega$, which is slightly larger than that of an impurity-free crystal $\left(R_{\square}=88.9 \Omega\right)$, indicating the existence of weak localization. In addition, the existence of weak Zn clusters may induce relatively wide superconducting channels and restrict phase-slipping, which will be much more pronounced in bulk crystals and relatively wide microbridges, as compared with mesoscopic system.

The impurity-free nanobridges demonstrated thermal stability due to smaller number of defects. With introducing $\mathrm{Zn}$, the nanobridges revealed several phase-slips. We propose that $\mathrm{Zn}$ suppresses superconductivity in a 3D 'Swiss cheese'-like pattern, where the order parameter is restricted to be developed along abundant narrow superconducting channels. Considering that the order parameter has zero value in the point of each impurity and since the magnitude of the order parameter can only change within its characteristic length scale, we can exclude the spherical regions with radius $\xi$ around each impurity from the superconducting condensate, for which the detail discussion on order parameter along the nanobridge is introduced in Supplementary Fig. 8 and Supplementary Note 2. We estimated the effective cross-section of the superconducting channels as a few nanometres according to the mechanism of thermally activated phase-slips. This cross-section value is consistent with the magnitude of the temperature-dependent coherence length supporting the proposed model.

For a conventional superconducting gap like $s_{++}$, the non-magnetic impurity ions work as point defects, but do not affect the Cooper pairs. Oppositely, the $\mathrm{Zn}$ ions can induce local destruction of superconductivity for the unconventional $s \pm$ pairing symmetry, and consequently result in phase-slip phenomenon in the $\mathrm{BKZn}$ nanobridges or even microbridges. The local destruction may provide an evidence for the pair-breaking effect of non-magnetic impurities and the unconventional $s \pm$ pairing symmetry for the iron pnictide superconductors. Meanwhile, the observation of an unusual large $A^{\star}$, especially for BKZn-N2, which could be due to the induced competing order around $\mathrm{Zn}$ impurities, suggests that further experiments might be necessary to achieve a better understanding of the nature of the superconducting gap symmetry in ironpnictides superconductors, as well as the role of $\mathrm{Zn}$ impurity.

\section{Methods}

Crystal growth. The $\mathrm{BK}\left(\mathrm{Ba}_{0.5} \mathrm{~K}_{0.5} \mathrm{Fe}_{2} \mathrm{As}_{2}\right)$ and $\mathrm{BKZn}\left(\mathrm{Ba}_{0.5} \mathrm{~K}_{0.5} \mathrm{Fe}_{1.94} \mathrm{Zn}_{0.06} \mathrm{As}_{2}\right)$ single crystals were grown using high-pressure technique as described elsewhere the stoichiometric mixture of BaAs, KAs, FeAs, Fe and $\mathrm{Zn}$ was placed in a tantalum capsule with an $h$-BN inner, and compressed at $3 \mathrm{GPa}$ in a belt-type high-pressure apparatus and heated at $1,300^{\circ} \mathrm{C}$ for $4 \mathrm{~h}$. The elemental concentration of BKZn was confirmed by energy dispersive X-ray spectroscopy (Supplementary Fig. 1).

Nanobridge fabrications. The single crystals were cleaved along their $c$ axis into flakes with thickness down to a few hundred nanometres, and then were glued onto Si substrates, with their $a b$ plane parallel to the substrate surface, by using a thin layer of epoxy. The crystals were then fabricated as microbridges as following process $^{10}$ : (i) Au depositing onto the crystal; (ii) annealing at $200{ }^{\circ} \mathrm{C}$ for $24 \mathrm{~h}$ under nitrogen atmosphere; (iii) photolithography patterning on the crystal; (iv) argon ion milling the sample into a microbridge; (v) removing the photoresist by acetone and connecting the electrodes with silver paste; and (vi) argon ion milling the whole sample to remove the Au layer. The thin crystals were patterned into microbridges with width $W=2 \mu \mathrm{m}$, length $L=10 \mu \mathrm{m}$ and thickness $h=100-400 \mathrm{~nm}$. Subsequently, some of the microbridges were cut by a focused ion beam system equipped with a Ga ion source (FEI Dual beam Strata 235) to produce constrictions within the bridges with $W$ down to $119 \mathrm{~nm}$ and different $L$. The focused ion beam milling was based on a procedure used earlier for YBCO thin films ${ }^{41}$. Here we present data on micro- and nanobridges with dimensions given in Table 1, where the nanobridges were patterned by ion milling with a focused Ga beam, while not for the microbridges. Figure la shows a scanning electron microscopy image of the nanobridge BKZn-N1. The thickness was confirmed from the resistance measurement of the microbridge ${ }^{10}$. 


\section{References}

1. Kamihara, Y. et al. Iron-based layered superconductor $\mathrm{La}\left[\mathrm{O}_{1-x} \mathrm{~F}_{x}\right] \mathrm{Fe} A s$ ( $x=0.05-0.12)$ with $T_{c}=26$ K. J. Am. Chem. Soc. 130, 3296-3297 (2008).

2. Paglione, J. \& Greene, R. L. High-temperature superconductivity in iron-based materials. Nat. Phys. 6, 645-658 (2010).

3. Mazin, I. I. Superconductivity gets an iron boost. Nature 464, 183-186 (2010).

4. Onari, S. \& Kontani, H. Violation of Anderson's theorem for the sign-reversing s-wave state of iron-pnictide superconductors. Phys. Rev. Lett. 103, 177001 (2009).

5. Anderson, P. W. Theory of dirty superconductors. J. Phys. Chem. Solids 11, 26-30 (1959).

6. Abrikosov, A. A. \& Gor'kov, L. P. Theory for the superconductivity of alloys. 1. Electrodynamics of alloys at absolute zero. Zh. Eksp. Teor. Fiz. 35, 1558 (1958; [Sov. Phys. JETP 8, 1090 (1959)].

7. Abrikosov, A. A. \& Gor'kov, L. P. Superconducting alloys at temperatures above absolute zero. Zh. Eksp. Teor. Fiz. 38, 319 (1959; [Sov. Phys. JETP 9, 220 (1959)].

8. Abrikosov, A. A. \& Gor'kov, L. P. Comment on 'Theory of impure superconductors: Anderson versus Abrikosov and Gorkov'. Phys. Rev. B 49, 12337 (1994).

9. Alloul, H. et al. Defects in correlated metals and superconductors. Rev. Mod Phys. 81, 45 (2009).

10. Li, J. et al. Superconductivity suppression of $\mathrm{Ba}_{0.5} \mathrm{~K}_{0.5} \mathrm{Fe}_{2-2 x} \mathrm{M}_{2 x} \mathrm{As}_{2}$ single crystals by substitution of transition metal $(M=\mathrm{Mn}, \mathrm{Ru}, \mathrm{Co}, \mathrm{Ni}, \mathrm{Cu}$, and $\mathrm{Zn})$. Phys. Rev. B 85, 214509 (2012).

11. Mazin, I. I., Singh, D. J., Johannes, M. D. \& Du, M. H. Unconventional superconductivity with a sign reversal in the order parameter of $\mathrm{LaFeAsO}_{1-x} \mathrm{~F}_{x}$. Phys. Rev. Lett. 101, 057003 (2008).

12. Sato, M. et al. Studies on effects of impurity doping and NMR measurements of La 1111 and/or Nd 1111 Fe-pnictide superconductors. J. Phys. Soc. Jpn 79, 014710 (2010)

13. Wang, Y. et al. Using controlled disorder to distinguish $s \pm$ and $s_{++}$gap structure in Fe-based superconductors. Phys. Rev. B 87, 094504 (2013).

14. Korshunov, M. M., Efremov, D. V., Golubov, A. A. \& Dolgov, O. V. Unexpected impact of magnetic disorder on multiband superconductivity. Phys. Rev. B 90, 134517 (2014).

15. Kontani, H. \& Onari, S. Orbital-uctuation-mediated superconductivity in iron pnictides: analysis of the five-orbital Hubbard-Holstein model. Phys. Rev. Lett. 104, 157001 (2010).

16. Kitagawa, S. et al. Nonmagnetic pair-breaking effect in $\mathrm{La}\left(\mathrm{Fe}_{1-x} \mathrm{Zn}_{x}\right)$ $\mathrm{AsO}_{0.85}$ studied by $\mathrm{As}^{75}$ and $\mathrm{La}^{139}$ NMR and NQR. Phys. Rev. B 83, 180501(R) (2011).

17. Pan, S. H. et al. Imaging the effects of individual zinc impurity atoms on superconductivity in $\mathrm{Bi}_{2} \mathrm{Sr}_{2} \mathrm{CaCu}_{2} \mathrm{O}_{8+\delta}$. Nature 403, 746-750 (2000).

18. Nachumi, B. et al. Muon spin relaxation studies of $\mathrm{Zn}$-substitution effects in high- $T_{c}$ cuprate superconductors. Phys. Rev. Lett. 77, 5421-5424 (1996).

19. Adachi, T. et al. Muon spin relaxation and magnetic susceptibility studies of the effects of nonmagnetic impurities on the $\mathrm{Cu}$ spin dynamics and superconductivity in $\mathrm{La}_{2-x} \mathrm{Sr}_{x} \mathrm{Cu}_{1-y} \mathrm{Zn}_{y} \mathrm{O}_{4}$ around $x=0.115$. Phys. Rev. B 69, 184507 (2004)

20. Yang, $\mathrm{H}$. et al. In-gap quasiparticle excitations induced by non-magnetic $\mathrm{Cu}$ impurities in $\mathrm{Na}\left(\mathrm{Fe}_{0.96} \mathrm{Co}_{0.03} \mathrm{Cu}_{0.01}\right)$ As revealed by scanning tunnelling spectroscopy. Nat. Commun. 4, 2749 (2013).

21. Yuan, H. Q. et al. Nearly isotropic superconductivity in ( $\mathrm{Ba}, \mathrm{K}) \mathrm{Fe}_{2} \mathrm{As}_{2}$. Nature 457, 565-568 (2009)

22. Zhang, L.-F., Covaci, L. \& Peeters, F. M. Position-dependent effect of non-magnetic impurities on superconducting properties of nanowires. Europhys. Lett. 109, 17010 (2015).

23. Little, W. A. Decay of persistent currents in small superconductors. Phys. Rev. 156, 396-406 (1967).

24. Tinkham, M. Introduction to superconductivity 2nd edn (Dover Publications, 2004).

25. Bezryadin, A. Superconductivity in nanowires (Wiley-VCH, 2013).

26. Skocpol, W. J. \& Tinkham, M. Fluctuations near superconducting phase transitions. Rep. Prog. Phys. 38, 1049 (1975)

27. Pekker, D. et al. Stochastic dynamics of phase-slip trains and superconductiveresistive switching in current-biased nanowires. Phys. Rev. B 80, 214525 (2009).

28. Giordano, N. Evidence for macroscopic quantum tunneling in one-dimensional superconductors. Phys. Rev. Lett. 61, 2137-2140 (1988).

29. Langer, J. S. \& Ambegaokar, V. Intrinsic resistive transition in narrow superconducting channels. Phys. Rev. 164, 498-510 (1967).
30. McCumber, D. E. \& Halperin, B. I. Time scale of intrinsic resistive fluctuations in thin superconducting wires. Phys. Rev. B 1, 1054 (1970).

31. Xu, K. \& Heath, J. R. Long, highly-ordered high-temperature superconductor nanowire arrays. Nano Lett. 8, 3845-3849 (2008).

32. Tian, M. et al. Observation of superconductivity in granular Bi nanowires fabricated by electrodeposition. Nano Lett. 6, 2773-2780 (2006).

33. Sahu, M. et al. Individual topological tunnelling events of a quantum field probed through their macroscopic consequences. Nat. Phys. 5, 503 (2009).

34. Bezryadin, A., Lau, C. N. \& Tinkham, M. Quantum suppression of superconductivity in ultrathin nanowires. Nature 404, 971-974 (2000).

35. Bonetti, J. A. et al. Electronic transport in underdoped $\mathrm{YBa}_{2} \mathrm{Cu}_{3} \mathrm{O}_{7-\delta}$ nanowires: evidence for fluctuating domain structures. Phys. Rev. Lett. 93, 087002 (2004).

36. Li, J. et al. Direct observation of the depairing current density in singlecrystalline $\mathrm{Ba}_{0.5} \mathrm{~K}_{0.5} \mathrm{Fe}_{2} \mathrm{As}_{2}$ microbridge with nanoscale thickness. Appl. Phys. Lett. 103, 062603 (2013).

37. Tinkham, M. et al. Hysteretic $I-V$ curves of superconducting nanowires. Phys. Rev. B 68, 134515 (2003)

38. Zhu, J.-X., Yu, R., Balatsky, A. V. \& Si, Q. Local electronic structure of a single nonmagnetic impurity as a test of the pairing symmetry of electrons in (K,Tl)Fe $\mathrm{Se}_{2}$ superconductors. Phys. Rev. Lett. 107, 167002 (2011).

39. Chen, H. et al. Disorder effects in multiorbital $s \pm$-wave superconductors: implications for $\mathrm{Zn}$-doped $\mathrm{BaFe}_{2} \mathrm{As}_{2}$ compounds. Phys. Rev. B 88, 184509 (2013).

40. Kleiner, R. \& Müller, P. Intrinsic Josephson effects in high- $T_{c}$ superconductors Phys. Rev. B 49, 1327-1341 (1994).

41. Schwarz, T. et al. Low-noise nano superconducting quantum interference device operating in tesla magnetic fields. ACS Nano 7, 844-850 (2013).

\section{Acknowledgements}

We thank Drs V.N. Gladilin, A.V. Silhanek, J. Fenton, H.-H. Wen, Z.-X. Shi, K. Jin and B.-Y. Zhu for valuable discussions. The work in KU Leuven was supported by Methusalem Funding by the Flemish government, the grants from Flemish Science Foundation (FWO) and the COST Action MP1201 from EU. Study in Nanjing University was supported by the National Natural Science Foundation of China $(11234006,51102188)$ and the Priority Academic Program Development of Jiangsu Higher Education Institutions. Researches in NIMS were supported by the World Premier International Research Center from MEXT, the Grants-in-Aid for Scientific Research $(25289233,25289108)$ from JSPS, and the Funding Program for World-Leading Innovative R\&D on Science and Technology (FIRST Program) from JSPS. X.K. and G.V.T. acknowledge the European Research Council, ERC Grant No 246791-COUNTATOMS.

\section{Author contributions}

J.L., H.-L.F., Y.-H.Y., K.Y. and E.T.-M. grew the single crystals. J.L., M.J., J.Y., Y.H., T.H. H.-B.W. and P.-H.W. fabricated the microbridges and measured transport properties. J.L., T.S., R.K. and D.K. cut the nanobridges using FIB. X.K. and G.V.T. measured the STEM-EDX and HAADF-STEM. J.L., G.Z., L.F.C., J.V. and V.V.M. measured the pulsed high fields and transport properties. J.L., M.J., J.Y., R.K., D.K., L.F.C., H.-B.W., J.V. and V.V.M. discussed the data. J.L., P.J.P. and L.F.C. proposed the model and simulated the results. J.L., D.K., R.K., H.-B.W. and V.V.M. analysed the data and prepared the manuscript.

\section{Additional information}

Supplementary Information accompanies this paper at http://www.nature.com/ naturecommunications

Competing financial interests: The authors declare no competing financial interests

Reprints and permission information is available online at http://npg.nature.com/ reprintsandpermissions/

How to cite this article: Li, J. et al. Local destruction of superconductivity by nonmagnetic impurities in mesoscopic iron-based superconductors. Nat. Commun. 6:7614 doi: $10.1038 /$ ncomms8614 (2015).

This work is licensed under a Creative Commons Attribution 4.0 International License. The images or other third party material in this article are included in the article's Creative Commons license, unless indicated otherwise in the credit line; if the material is not included under the Creative Commons license users will need to obtain permission from the license holder to reproduce the material. To view a copy of this license, visit http://creativecommons.org/licenses/by/4.0/ 